\title{
Data-driven energy management system with Gaussian process forecasting and MPC for interconnected microgrids
}

\author{
Leong Kit Gan, Member, IEEE, PengFei Zhang, Jaehwa Lee, Michael A. Osborne, and David A. \\ Howey, Senior Member, IEEE
}

\begin{abstract}
Interest in predicting and optimising microgrid operation with a high proportion of variable renewable energy generation is growing. In this paper, we study and experimentally analyse the performance of a Gaussian-process regression forecasting and model predictive control algorithm in the context of interconnected microgrids. The scheme, which operated at six hours time horizon, achieved superior results with only a small deviation from the optimal operation calculated offline assuming perfect foresight. We also demonstrate that whilst a longer horizon provides a better solution in terms of lower cost of electricity, the battery cycling rate is also higher. Finally, we demonstrate improvements in renewable and load forecasts by sharing information between the microgrids.
\end{abstract}

Index Terms-microgrids, battery, energy management systems, predictions, MPC, optimisation

\section{INTRODUCTION}

$\mathrm{I}_{\mathrm{o}}^{\mathrm{N}}$ NTEREST in microgrids has increased significantly since they offer a resilient and scalable option for integrating dispatchable generation, non-dispatchable generation, energy storage systems and loads. Recently, networked or interconnected microgrids are also gaining attention and could help to address the congestion issue on existing grid infrastructure [1]. Effectively coordinating and optimising the performance of many microgrids is non-trivial and requires further research into advanced energy management system (EMS) algorithms.

Typically, an EMS operates at a lower bandwidth in comparison with the voltage, current and other control loops within power electronics converters. The former, also known as tertiary control, attempts to optimally balance supply and demand over longer time intervals. As part of an advanced EMS algorithm, it is common to predict renewable generation resources, load demands, and time of use tariff profiles subjected to uncertainties [2]. The EMS may individually or collectively consider the economic, technical or environmental constraints depending on the types of load and services provided by the particular microgrid.

In the past, EMS research was notably focused on the formulation of constrained rule-based strategies [3, 4] whilst recent advances in machine learning techniques have motivated researchers in this field to work on predictive strategies implemented by optimisation $[5,6]$, which are more realisable due to the presence of increasingly powerful microprocessors. Nonetheless, limited literature can be found on the coupling between an optimisation strategy and a forecasting algorithm, especially for an online system. Whilst many investigators have formulated more computationally efficient optimisation algorithms, they do not routinely consider the required processing time for forecasting algorithms, which may ultimately constrain real-time execution.

It is also widely acknowledged that the uncertainties associated with a predictive algorithm are important when assessing the robustness of an EMS. A common way to emulate uncertainties is by adding a fixed noise term [7], which may be regarded as an oversimplification. On the other hand, inappropriate selection of a predictive model may reduce the performance of an EMS.

\section{A. Literature review}

Various strategies can be considered in formulating an EMS for a network of microgrids. Intuitively, energy cooperation among microgrids may reduce the cost of electricity $(\mathrm{CoE})$ and local energy storage system (ESS) capacity required. This is because when net energy profiles are complementary between sites, it is likely that energy surplus at one site will compensate for energy deficit elsewhere [1]. Two EMS algorithms were derived; namely the store-then-cooperate approach and the cooperate-then-store approach. The choice between these options relies on the distance between the microgrids, the correlation coefficient between the microgrids' net energy profiles, their ESS capacities, etc. [1]. In another work [8], a priority-based energy scheduling method was proposed as a non-pricing-based mechanism devised for a real-time implementation in the actual electricity market. Luna et al. [9] quantified the effectiveness of a comprehensive online EMS and identified that significant improvements can be made, especially with respect to forecasting algorithms.

Model predictive control (MPC) is recognised as a practical approach for solving energy dispatch problems [10-12]. Reoptimisation at each time step compensates for the changes of variable renewable sources or load demands. Researchers in [5] further extended this method to manage power flow within a networked microgrid. To minimise system frequency and tieline power flow deviations, an MPC-based anticipatory frequency control used within the conventional Automatic Generation Control framework was proposed [13]. Separately, 
the authors in [14] used MPC to optimise the operation of a microgrid with constraints associated with a hybrid ESS that included ultracapacitors, batteries, and hydrogen storage. The work was further extended to an interconnected microgrid with distributed MPC [15]. It was found that networked operation of microgrids can improve the economic benefits whilst maximising ESS lifetime. Similarly, distributed MPC for interconnected microgrids has been used for energy trading [16], and mitigation of outages in island systems has been implemented using MPC to estimate the battery stored energy within a certain bound and executed pre-emptive non-critical load shedding [17]. Stochastic optimisation and stochastic MPC has also been explored for EMS operation [18] and is a useful technique for accommodating the uncertainties of load demand, renewable generation and price [19]. A transactive energy system for several interconnected microgrids was demonstrated to minimise voltage deviations [20]. Dynamic pricing established through a market process organised by the DNO could also be an effective technique for distributed optimal control of microgrids [21].

In terms of forecasting algorithms, traditional parametric regression methods may not achieve good prediction accuracy [22]. In contrast, non-parametric regression could extrapolate better when the random variable is non-stationary [23]. In this work, we use Gaussian Processes (GPs) for predicting solar PV generation, and load demand. GPs have been proven to be effective non-parametric models for stochastic regression. They have been widely used [24, 25] to predict wind power and solar power generation. In contrast to parametric regression (e.g. linear regression), GPs can achieve better estimation accuracy, measured by the root mean square error (RMSE) [26]. GPs require minimal parametrisation and they are purely data-driven based on correlations between future data points and historical data points. In comparison with methods such as neural networks, GPs does not require as much training data. Accuracy of forecasting solar PV generation using data-driven techniques may be improved by considering correlated data streams from more than one geographically distributed site [27]. GPs are able to handle this kind of spatially distributed data in an intuitive way, as we shall explore later in this paper.

\section{B. Main contributions}

In this paper, we study and demonstrate control of interconnected microgrids, adopting GP time-series modelling for predicting the energy generation and load demand, followed by optimising the power flow using MPC. The closely coupled and frequent updates of forecast and optimisation are able to minimise the forecast error and whilst at the same time reducing the discrepancy [28] of battery state-of-charge (SoC) estimated from the battery management system (BMS) vs. EMS respectively. In our work, GP forecasts are computed locally at each distributed computing unit, thereby reducing the computational effort required by the centralised controller. The underlying physical constraints (e.g. Kirchhoff's laws) which influence the power flow among the interconnected microgrids [29] were expressed using an optimal power flow (OPF) scheme [30, 31]. The overall framework requires a mixed integer linear program (MILP) and in this case we adopted the commercial CPLEX solver from IBM [32]. It is acknowledged that in a much larger practical system, computational load for solving MILPs increases rapidly with the number of integer constraints, where infeasible solutions may be encountered. Besides decentralised algorithms, a hybridised centraliseddecentralised approach may be used to coordinate as the number of microgrids scales up.

By varying the MPC horizon, we explored the trade-off between $\mathrm{CoE}$ purchased from the utility and the degree of battery utilisation (which uses more renewables). The prediction accuracy affects the overall economic benefits; hypothetically, better accuracy should produce a solution closer to optimum. In view of this, we propose the sharing of information (in this case PV power generation and load demand) among the microgrids which are co-located within a certain region to improve their prediction accuracy; this novel approach is called a co-regionalised GP (CoRGP), and exploits correlations between spatial locations. CoRGP improves prediction accuracy and mitigates the impact of missing data [33], but may require more computational resources.

Rather than only performing simulations, our proposed GPMPC scheme for interconnected microgrids was experimentally validated. The interaction and latency between the highsampling rate BMS and the low-sampling rate EMS is also taken into consideration [28]. We considered horizon lengths of less than six hours for practical implementation. Unlike most of the previous work on EMS's, we included physical constraints in the model so that physical limits were not violated. Our laboratory-scaled experiments also had realistic behaviour in terms of control, communication, measurements, and dynamics. We tested the algorithm using realistic PV power and load demand profiles with different MPC horizon lengths, followed by a comparative analysis on $\mathrm{CoE}$ purchased from the utility, and the corresponding battery utilisation.

\section{Modelling OF THE GP-MPC}

Fig. 1 describes the overall operation of the proposed EMS scheme. It uses a GP or CoRGP to predict PV power and load demand, followed by the MPC, which minimises the objective function subject to the constraints over the considered horizon. Initialisation takes place at the beginning by setting all the batteries to idle. If the EMS operation is enabled, the local supervisory control and data acquisition (SCADA) system of each microgrid gathers appropriate sets of measurement (PV power and load demand) and predict the values for next horizon. Once predictions are completed and using the latest measured battery SoC values, MPC proceeds in finding the optimal solutions (battery power set-points) for each converter whilst respecting all constraints. These set-points are then sent to all the microgrids and handled by the local BMS. The cycle repeats indefinitely as long as the EMS operation is not aborted and the SCADA system receives PV and load predictions from each microgrid. The following sections explain the GP and MPC modelling respectively. 


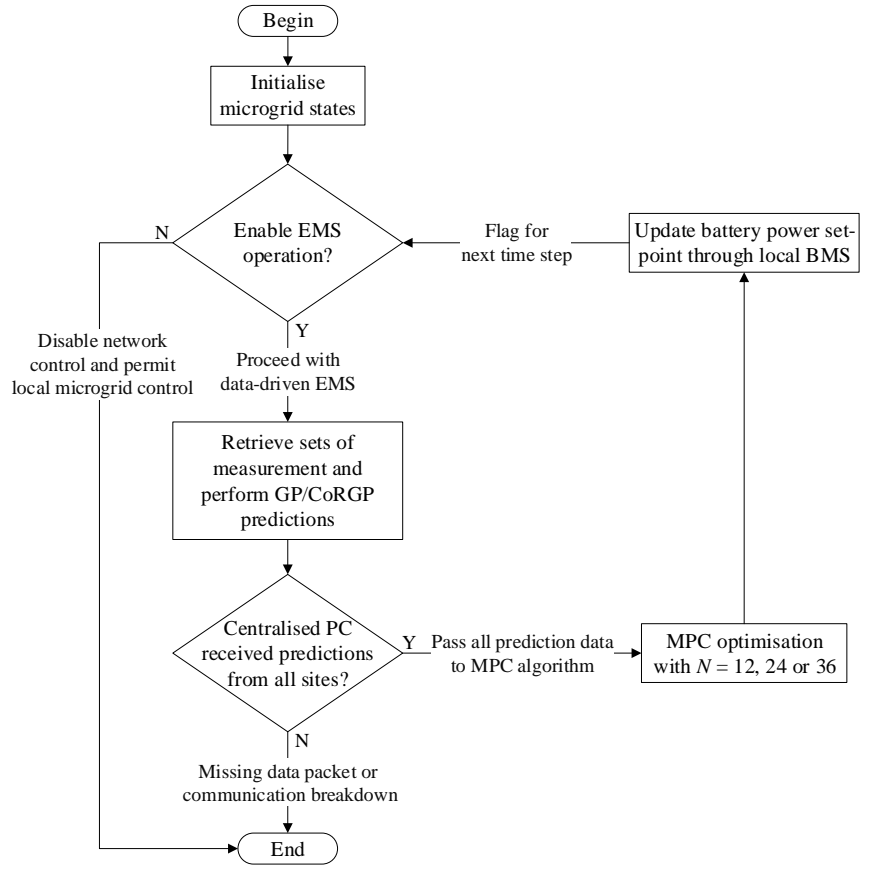

Fig. 1. Overall framework of the proposed GP-MPC scheme, updated at every sampling time, $T_{\mathrm{s}}$

\section{A. GP for time-series modelling}

The section provides a formal definition of GP and CoRGP respectively. Specifically, they are derived for time-series modelling.

1) Gaussian processes. GP regression is a Bayesian regression method for non-linear, stochastic, complex problems. We start with the general definition of Gaussian process (GP).

Definition 1: Gaussian process. Denote by $\mathrm{f}(\mathrm{x}, \mathrm{t}) .: \mathcal{X} \mapsto \mathbb{R}$ a stochastic process parameterized by $\{x, t\} \in \mathcal{X}$, where $X \in$ $\mathbb{R}^{2} \times \mathbb{R}^{+}$. Then, the random function $\mathrm{f}(x, t)$ is a Gaussian process if all its finite dimensional distributions are Gaussian, where for any $m \in \mathbb{N}$, the random variables $\left(\mathrm{f}\left(x_{1}, t_{1}\right), \cdots, \mathrm{f}\left(x_{\mathrm{m}}, t_{\mathrm{m}}\right)\right)$ are jointly normally distributed. The model assumes that data follows a multivariate Gaussian distribution where the mean and covariance between any two points are specified by the mean function and covariance function respectively. We can interpret a GP as formally defined by the following class of random functions:

$$
\begin{aligned}
& \mathcal{F}:=\{\mathrm{f}(\cdot): \mathcal{X} \mapsto \mathbb{R} \\
& \text { s.t. } \mathrm{f}(\cdot) \sim \mathcal{G} \mathcal{P}(\mu(\cdot ; \theta), \mathcal{C}(\cdot ; ; \Psi)), \text { with } \\
& \mu(\cdot ; \theta):=\mathbb{E}[\mathrm{f}(\cdot)]: \mathcal{X} \mapsto \mathbb{R}, \\
\mathcal{C}(\cdot ; ; \Psi):= & \left.\mathbb{E}\left[(\mathrm{f}(\cdot)-\mu(\cdot ; \theta))(\mathrm{f}(\cdot)-\mu(\cdot ; \theta))^{T}\right]: \mathcal{X} \times \mathcal{X} \mapsto \mathbb{R}^{+}\right\}
\end{aligned}
$$

2) Priors. Before measurement, at each point the (prior) mean of the function is $\mu(\cdot ; \theta)$ parameterized by $\theta$, and the (prior) spatial dependence between any two points is given by the covariance function (Mercer kernel) $\mathcal{C}(\cdot, ; \Psi)$, parametrized by $\Psi$, more detailed discussion can be found in Rasmussen and Williams [34]. Then, CoRGPs were developed by Neil to study multi-output GPs [35].

3) Definition 2: CoRGP. Denote by $f_{1}(x, t)$, $f_{2}(x, t), \cdots, f_{n}(x, t),: \chi \mapsto \mathbb{R} n$ correlated stochastic processes parameterized by $\{x, t\} \in \mathcal{X}$, where $\mathcal{X} \in \mathbb{R}^{2} \times \mathbb{R}^{+}$. Then, the random function $f_{i}(x, t)$ where $i=\{1,2, \ldots n\}$ is a CoRGP if each $f_{i}(x, t)$ is drawn from a GP and any two $f_{i, j}(x, t)$ are correlated with values $K_{i j}$ where

$$
K(\mathrm{i}, \mathrm{j} ; \Psi):=\mathbb{E}\left[\left(f_{i}(\cdot)-\mu_{i}\left(\cdot ; \theta_{i}\right)\right)\left(f_{j}(\cdot)-\mu_{j}\left(\cdot ; \theta_{j}\right)\right)^{T}\right]
$$

The discrete kernels describe the correlation amongst the PV generation and load demand respectively. As an example, if there are $N$ number of sensors for PV generation, the particular discrete kernel specifies the correlation between pair wise nodes, i.e. elements at row $i$ and column $j$ in the matrix reflect the correlation between PV panel $i$ and PV panel $j$. So, in total there will be $N^{*} N / 2-N$ parameters to be determined for this discrete kernel. Same principle applies for load demand.

\section{B. $M P C$ for interconnected microgrids}

The fundamental principle of MPC in our work is that at each time step, a finite horizon optimal control is computed for the battery power and power exchanged with the utility. However, only the first time-step of the control action is executed. The algorithm operates in a receding rolling horizon manner where at the next discrete step $(k+1)$, the optimal solution over the next $N$ periods is computed based on the new predicted renewable generation and load demands. In our work, the MPC controller is implemented using the following steps:

1) At $k=1$, the states are initialised from the first measurement, i.e. batteries SoC, PV power generation, load demand, power exchange with the utility and its associated energy price

2) Using the PV power and load demand predictions from GP or CoRGP, the algorithm computes an optimal control sequence for the rolling optimization horizon $(N)$ at sampling time, $T_{\mathrm{s}}$ of every 10 minutes

3) Execute the first control period operation of the scheduling problem

4) Update the information available in each microgrid (in this case the battery power set-point) for the next period. Then, move on to the next sampling instant and repeat the algorithm.

The controller aims to minimise the sum of net electricity cost over the scheduling horizon, as written in equation (2). The variables $C_{\mathrm{g} \text {, imp }}$ and $C_{\mathrm{g}}$, exp respectively represent the import and export unit energy price from the utility, whilst $P_{\mathrm{g}, \text { imp }}$ and $P_{\mathrm{g} \text {, exp }}$ respectively represent the import and export power exchanged with the utility. The battery $\mathrm{SoC}$ for each microgrid, $S o C_{\mathrm{m}}$ is estimated with the coulomb counting approach, as shown in equation (3). $Q$ represents the battery capacity in microgrid $m$ in Watt-hours. $\eta_{\mathrm{c}}$ and $\eta_{\mathrm{d}}$ denote the battery charging and discharging efficiencies respectively. Equation (4) sets the upper and lower limits for discharging $\left(P_{\mathrm{batt}, m}^{\mathrm{max}}\right)$ and charging $\left(P_{\mathrm{batt}, m}^{\min }\right)$ power respectively. In each microgrid, the battery SoC is maintained between the limits (equation (5)) of $30 \%$ $\left(S o C_{m}^{\min }\right)$ and $80 \%\left(S_{m} C_{m}^{\max }\right)$. Note that we used a scaled-down DC microgrid testbed (described in section IIIA) to emulate the actual power grid for experiment purposes. Since the focus here is to evaluate the proposed EMS algorithm, either an AC or a DC system may be used for validation. The classical OPF principle is used in this work. If an unbalanced distribution system is considered, the algorithm is still applicable by having 
the batteries interface with three units of single-phase inverters and the energy is aggregated among them. The computed power set-point for that particular node can then be divided with appropriate ratio for each inverter. However, for simplicity, this work assumes a balanced microgrid system.

Conventionally, the classical OPF approach [31] is used to find the minimal cost of generation that respects the voltage and thermal (line flow) constraints. Once generation and demand levels are set, the physics determines the line flows and hence, additional constraints are necessary to model this. To achieve fast computation, the linearised DC OPF was derived from the AC OPF under three assumptions: i) the reactance of each line is greater than its resistance, ii) the voltage magnitude at each bus is at the nominal level, iii) the voltage phase difference between adjacent buses is small and thus the real power on a line is proportional to the voltage phase difference along the line, and there is no reactive power flow [31]. We adopted the DC OPF in our work, where the Kirchhoff voltage law, expressed in equation (6) determines the power flow $\left(f_{l}(k)\right)$ between microgrid buses as a function of voltage phases $\left(\delta_{m}(k)\right)$ at both ends. Elements $a_{m l}$ take the values -1 if $m$ is the start bus of $l$ and +1 if $m$ is the end bus of $l ; x$ is the reactance of the line. This equation assumes that the power losses due to electrical resistance are neglected. The voltage phases are uniquely determined (decision variables) and the utility bus is chosen as the reference bus with zero voltage phase, $\delta_{\text {grid }}=0$. Since the utility grid is connected to microgrid 2 and the power trading is limited, we introduce $\delta_{2}^{+}$ and $\delta_{2}^{-}$such that $\delta_{2}=\delta_{2}^{+}+\delta_{2}^{-}$with $\delta_{2}^{+} \geq 0$ and $\delta_{2}^{-} \leq 0 . \delta_{2}^{+}$ and $\delta_{2}^{-}$are treated as special ordered sets of type 1 (SOS1) variables [36], so that at most one of them can be assigned nonzero. The Kirchhoff current law shown in equation (7) ensures the conservation of power flow within a microgrid is respected. Note that the PV power, $\hat{P}_{\mathrm{PV}, \mathrm{m}}(k)$ and load demand, $\hat{P}_{\text {load, } \mathrm{m}}(k)$ are the predicted values from GP or CoRGP. The battery power $P_{b a t t, m}$ is then formulated as the sum of charge and discharge power for each microgrid, $P_{\text {batt }, m}(k)=P_{\text {batt }, m}^{D}(k)+$ $P_{\text {batt }, m}^{C}(k)$, where $P_{\text {batt }, m}^{D}(k)$ and $P_{\text {batt }, m}^{C}(k)$ are also formulated as SOS1 variables. We defined the manipulated variables as $U(k)=\left[\delta_{2}^{+}(k), \delta_{2}^{-}(k), P_{b a t t, m}^{D}(k), P_{\text {batt }, m}^{C}(k)\right]^{T}$ for conciseness.

$$
\min _{U(0), U(1), \ldots, U(N-1)} \sum_{k=0}^{N-1}\left(C_{g, \text { imp }}(k) \cdot P_{g, \text { imp }}(k)-C_{g, \text { exp }}(k) \cdot P_{g, \text { exp }}(k)\right)
$$

Subject to:

$$
\begin{aligned}
& S o C_{m}(k+1)=\left\{\begin{array}{l}
S o C_{m}(k)-\eta_{c} \frac{T_{s}}{Q_{m}} P_{\text {batt }, m}(k), \text { if } P_{\text {batt }, m}(k) \leq 0 \\
S o C_{m}(k)-\eta_{D} \frac{T_{s}}{Q_{m}} P_{\text {batt }, m}(k), \text { if } P_{\text {batt }, m}(k) \geq 0
\end{array} \forall m \in M\right. \\
& P_{b a t t, m}^{\min } \leq P_{b a t t, m}(k) \leq P_{b a t t, m}^{\max } \quad \forall m \in M \\
& \mathrm{SoC}_{m}^{\min } \leq \operatorname{SoC}_{m}(k) \leq S o C_{m}^{\max } \quad \forall m \in M \\
& f_{l}(k)=-\frac{V_{0}^{2}}{x_{l}} \sum_{m \in M} a_{m l} \delta_{m}(k) \quad \forall l \in L, \forall m \in M \\
& \hat{P}_{P V, m}(k)+P_{\text {batt }, m}(k)+\sum_{l} a_{m l} f_{l}(k)=\hat{P}_{l o a d, m}(k) \quad \forall m \in M(7)
\end{aligned}
$$

\section{EVAluation OF The PRoposed GP-MPC EMS ALGORITHM LABORATORY SETUP}

In this section, a brief description of our laboratory-scaled interconnected microgrid test platform is given. Then, comparison between GP and CoRGP predictions of PV and load demand with a 6-hour horizon is demonstrated. Using the set of parameters defined in this work for the GP and MPC algorithms, the overall performance and interactions between the microgrids and the utility grid are analysed.

\section{A. Laboratory-scaled interconnected microgrids}

We used three interconnected microgrid emulators to test the proposed EMS. The microgrid system block diagram is shown in Fig. 2 [28] and the corresponding hardware setup is shown in Fig. 3 [28]. The microgrids can communicate with each other via TCP/IP, enabling distributed forecasting, control, and optimisation. Although it is a scaled-down microgrid system, it nonetheless is realistic in terms of control interfaces, measurements, and dynamics, and therefore provides a valuable insight into EMS implementation which cannot be obtained through simulations alone. For simplicity, we adopted a centralised EMS approach. In this configuration, the utility grid is connected to microgrid 2 whilst the other microgrids are power linked with each other in a ring topology.

The adopted microgrid configuration is a dc system, which has the advantage of not requiring synchronisation, frequency and phase control devices. The PV emulator and lead-acid battery were connected to the common dc bus via bespoke bidirectional half bridge dc-dc converters. For simplicity, a power supply and an electronic load were used to emulate the utility grid. The power supply was programmed to control the voltage of the common dc bus and to supply the power deficit within

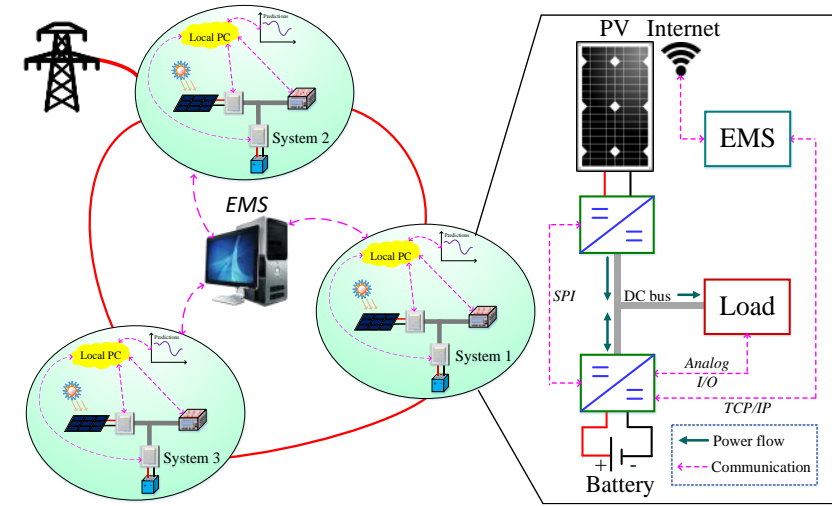

Fig. 2. Block diagram of the laboratory-scaled networked microgrids [28]

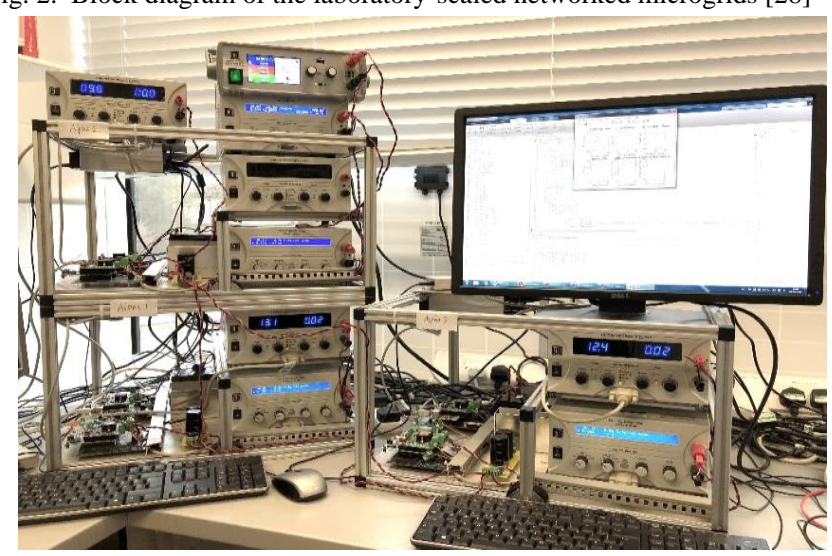

Fig. 3. Interconnected microgrid emulators in the lab [28] 

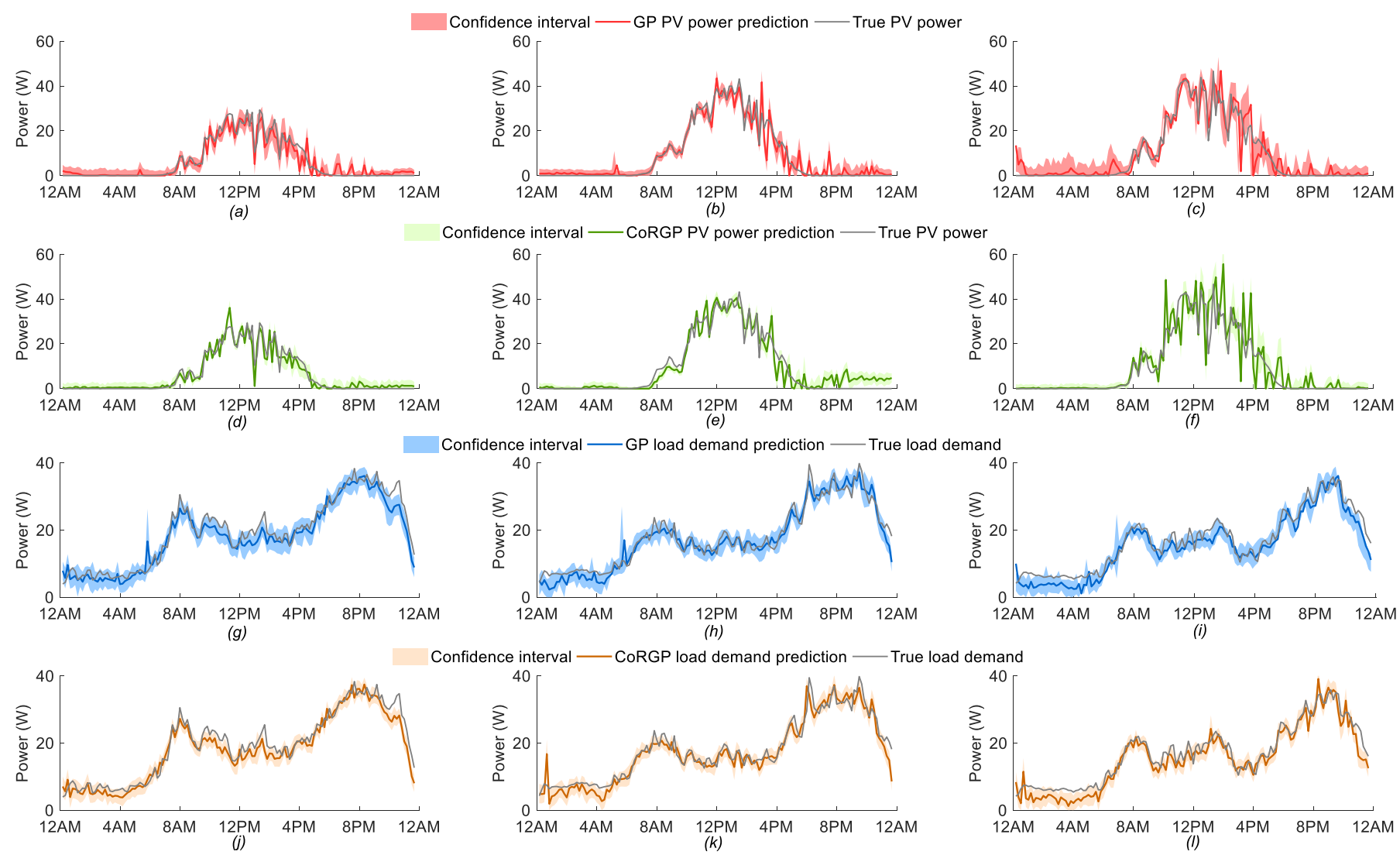

Fig. 4. Six-hour rolling horizon GP PV power predictions for (a) microgrid 1, (b) microgrid 2 and (c) microgrid 3. CoRGP PV power predictions for (d) microgrid 1 , (e) microgrid 2 and (f) microgrid 3. GP load predictions for (g) microgrid 1, (h) microgrid 2 and (i) microgrid 3. CoRGP load predictions for (j) microgrid 1, (k) microgrid 2 and (l) microgrid 3.

the microgrid system. In terms of software structure, a low-level program, implemented in an ARM-based microcontroller (STM32F401RET6), executes the control logic and establishes communication links between the EMS algorithm and hardware. The low-level logic mainly controls the dc-dc converters, provides a current reference for the electronic load, implements the battery management system, and protects the microgrid. The next layer (the EMS) is run within MATLAB on a PC. More details on the hardware and software implementation of the microgrid can be found in [37].

\section{B. GP and CoRGP predictions}

The measured PV and load profiles were averaged over 10 minutes and fed as inputs to the GP models. The original 1minute resolution profiles were used as control inputs for the PV emulator and electronic load. As an example, the six-hour horizon GP and CoRGP predictions for both PV generation and load demand are shown in Fig. 4. Note that in this case, only the first ten-minute prediction point over each six-hour horizon is shown and hence 144 data points for one day is plotted. We plotted the mean as well as the one standard deviation (i.e. $68.27 \%$ confidence interval) for each prediction. The CoRGP could achieve high forecasting accuracy compared to the normal GP models. As explained, the CoRGP uses information from other nearby microgrids to improve its predictions.

\section{Analysis of the microgrid operation}

In this section, the local GP prediction for the six-hour horizon scenario is selected for a more detailed discussion and analysis. The parameters are listed in Table I and Fig. 5 shows the experimental measurements. It is acknowledged that a longer operation (for e.g. one month) may provide a better test coverage. However, such analysis is more appropriate to be carried out using simulation tools where the run-time is accelerated [38]. In addition, since the solar irradiance and load demand profiles are periodic in nature, therefore, the accumulative savings over a longer period may simply be aggregated. If on the other hand more random profiles were considered, for example wind energy, a longer experiment may be more beneficial. Nonetheless, in this work, high resolution solar irradiance and load demand profiles were used and they were different amongst the microgrids, hence the variations and dynamics were taken into consideration. The PV power from

TABLE I

GP-MPC EXPERIMENTAL SPECIFICATIONS

\begin{tabular}{l|c}
\hline \multicolumn{1}{c}{ Parameters } & Values \\
\hline Horizon & 6 hours \\
GP/CoRGP for prediction & GP \\
Training length & 2 days \\
GP iterations & 10 \\
Sampling time & 10 minutes \\
Battery SoC boundary & $30 \leq \mathrm{SoC} \leq 80$ \\
Battery capacity & $120 \mathrm{Wh}$ \\
Battery charging efficiency & $95 \%$ \\
Battery discharging efficiency & $96 \%$ \\
Maximum and minimum battery power & $\pm 45 \mathrm{~W}$ \\
Maximum and minimum grid power & $\pm 131.25 \mathrm{~W}$ \\
Import electricity tariff (12 am to $7 \mathrm{am})$ & $0.0071 \mathrm{unit} / \mathrm{Wh}$ \\
Import electricity tariff $(7$ am to $12 \mathrm{pm})$ & $0.0152 \mathrm{unit} / \mathrm{Wh}$ \\
Transmission line resistance & $0.01 \Omega$ \\
\hline
\end{tabular}




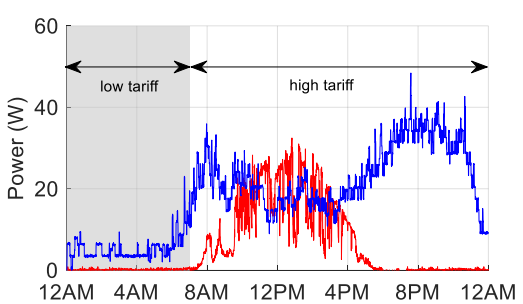

(a)
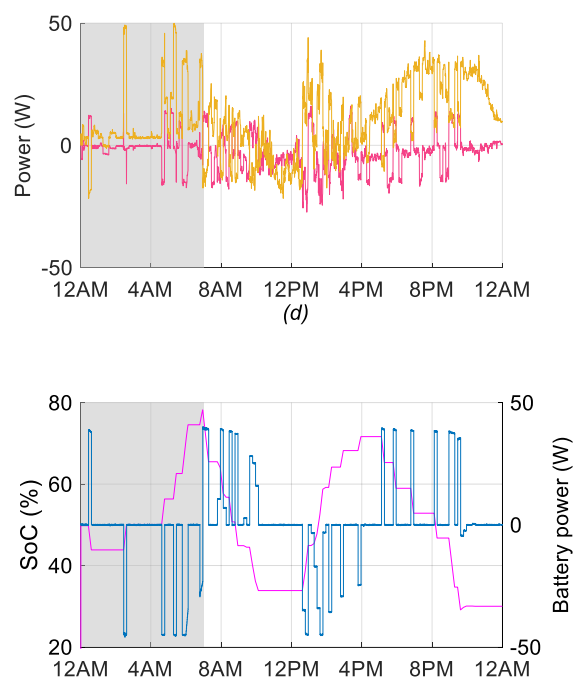

(g)

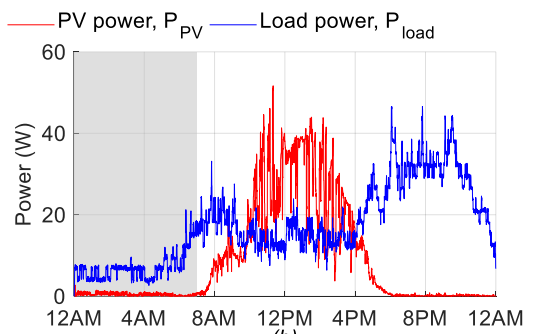

(b)
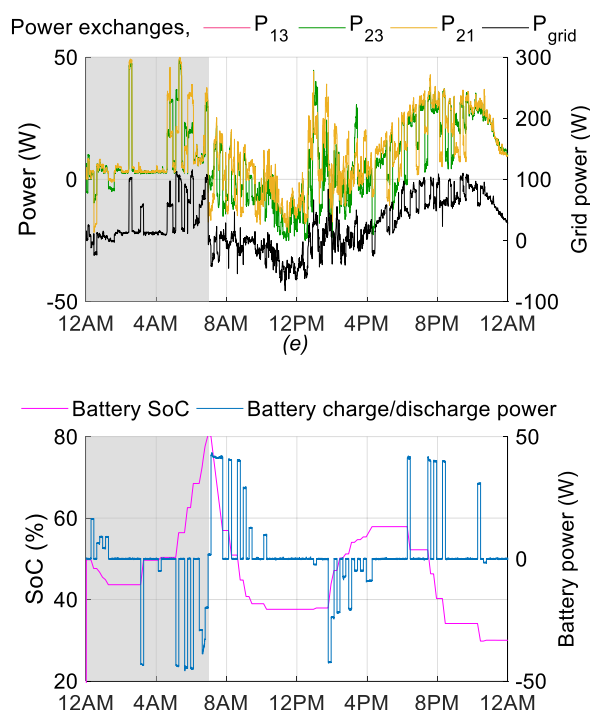

(h)

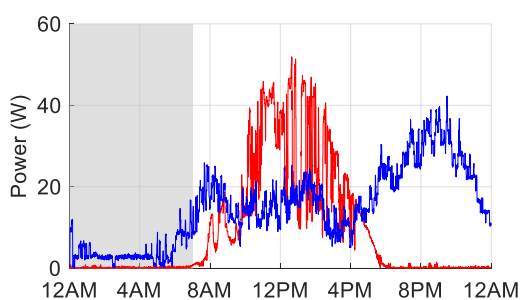

(c)
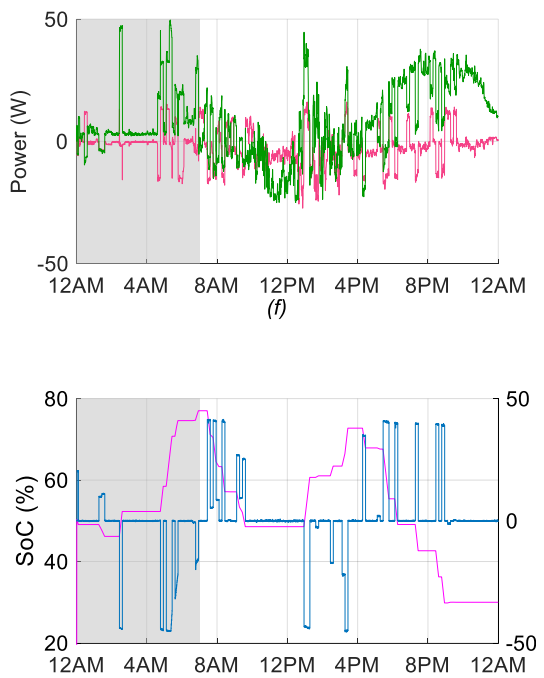

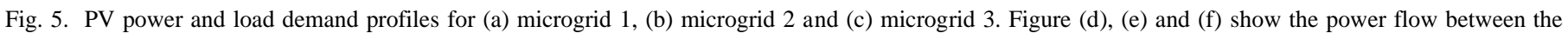
microgrids. The battery charge/discharge and its corresponding SoC profiles for (g) microgrid 1, (h) microgrid 2 and (i) microgrid 3

microgrid 1 was lower than the other two sites whilst the loads for all sites were relatively similar in magnitude.

Two electricity tariffs from the utility were considered in this case study. Between 12 am and 7 am (background filled in grey), the price was almost half of the higher tariff which was between 7 am to 12 midnight. In addition, the price of selling electricity to the grid was half of that for buying from the grid at all times. Using the GP predicted PV power and load demand with the electricity tariff over the next six hours, the MPC optimised the operation of the networked microgrids whilst ensuring the grid and battery constraints were not violated. Again, the objective function was to minimise the CoE.

Just before the electricity tariff became more expensive, the batteries were charged close to the upper limit (80\% SoC) as the EMS foresaw that there would be an energy deficit situation at later times. In this case, the EMS decided to charge the batteries at around $4.30 \mathrm{am}$. More power transfer was observed between microgrids 2 and 3, and between microgrids 2 and 1, due to connection of microgrid 2 with the utility grid. However, minimal power flow took place between microgrids 1 and 3 due to their similar load and PV profiles in the morning. Note that although the EMS optimised the operation for the next six hours, only the first battery set-point was used and there was no gradient imposed on the cost function over the horizon. This means no priority was given to any of the set-point solutions.

In the middle of the day, when there was electricity surplus from the PV panels, it should either be stored locally, used to supply the loads from other microgrids or exported to the utility grid. Between about 10 am to $1 \mathrm{pm}$, the excess electricity was mainly exported to the grid because the EMS was not able to foresee the shortage in PV generation in the evening (due to the 6-hour horizon), thus generating income for the consumers. When the EMS noticed that electricity deficit would occur in the evening, storing the renewable surplus locally began to take place from about $1 \mathrm{pm}$ onwards until about $4 \mathrm{pm}$ where the surplus of electricity tapered off. In this case, the battery SoC upper limit was not reached due to the limited horizon (six hours) foreseeable by the EMS. On the contrary, if the predictive horizon was longer, the battery capacity would be better utilised to store the PV surplus without aiming to gain short-term monetary value (which is half of the purchase electricity price) by exporting the electricity to utility grid. Nevertheless, this assumes that the forecast algorithm performs well with longer horizons which would otherwise be contradictory to the earlier optimal decisions. For instance, if the load were much lower than the expected values in the evening and it turned out that the electricity export occurred between 12 am and 7 am, the profit would be lower. In essence a limited horizon strategy, besides requiring less computational effort, may be viewed as reducing the risk of incurring more electricity cost due to inaccurate predictions downstream, however this comes with the expense of being less optimal overall.

As the energy deficit phenomena continued through the evening, electricity was purchased from the utility whilst the batteries were gradually discharged until they reached the lower limit of $30 \%$ SoC. In other words, the EMS was attempting to achieve the lowest possible $\mathrm{CoE}$ by using up all the PV energy stored earlier. Whilst the CoE may be minimised, it is important to note that the battery degradation cost was not incorporated in 
the EMS in this case. Intuitively, if the battery degradation cost of using the batteries is smaller than the utility CoE, the operation may remain similar due to the economic benefit. On the other hand, the batteries may not be used at all if the $\mathrm{CoE}$ from the utility is cheaper than the battery penalty cost.

\section{Discussion of market framework}

The modern electricity market can be categorised into different time-scales; the real-time balancing market, day-ahead market and hour-ahead market. A real-time balancing market is triggered at each sub-hourly interval (e.g. 5-15 minutes) during the operational hour. The authors in [39] proposed a hierarchical real-time balancing market where clusters of microgrids under the management of a distribution system operator (DSO) each bid to provide balancing services. Since the DSO does not own any of the power generation and storage assets, it stimulates the local microgrids to reach the cleared market bid by releasing a price signal, i.e. the distribution locational marginal price [39]. Such a market framework can integrate our proposed predict-then-optimise control strategy (10 minutes sampling time) which falls under the category of sub-hourly interval. For instance, the microgrids can be aggregated as a single entity and participate in the balancing services (such as fast reserve and short-term operating reserve) with the DSO.

With the advancement in computing capability which enables prediction and optimisation to be completed in a shorter time-frame, the microgrids will be in a better position to participate in frequency response and voltage control services. These services are important to ensure system stability as the integration of renewable generation increases. In addition, the higher sampling rate may also reduce the state error (in this case the battery SoC) due to a more frequent state update. Indeed, the trade-off sensitivity in terms of computational time, optimality which relates to prediction accuracy and the revenues resulted from a stack of grid services is an important consideration for practical implementation.

\section{SENSITIVITy ANALYSIS}

In this section, we aim to compare and analyse the microgrid performances with GP and CoRGP predictions across different horizon lengths. The offline scenario with perfect PV power and load demand forecasts serves as the benchmark case [18, 40]. In theory, as the prediction accuracy improves, the optimisation algorithm should produce a better solution with a lower CoE. We tabulated the sum of both PV and load absolute error $\left(\sum_{i=1}^{144}\left|y_{\text {pred }}-y_{\text {true }}\right|\right)$ between the mean of predicted and true values in Table II for two, four and six hours horizons. Due to the limited computational power of the PC used in completing the GP iterations within the sampling time (i.e., 10 minutes), longer horizons were not considered in this study.

In general, the computed errors for CoRGPs are smaller than the GPs. In terms of the accumulated prediction errors for the first sample, the CoRGP always outperforms the GP. This is because the CoRGP uses the data from all three systems for predictions. Table II shows the average errors associated with the considered scenarios. We also found that when more training samples were used (for the CoRGP case), the prediction error reduced, but with longer computational time. Therefore, there is a tradeoff between the prediction error and the amount of training data. One way to reduce the prediction time is to use fewer training samples or reduce the number of iterations in the optimisation steps. It is also noticed that the largest error for the CoRGP cases occurred at four hours prediction length, whereas the error decreased as the horizon increases for the GP scenarios. This presents an interesting phenomenon where the parameters (e.g. training length, iterations etc) may need to be further optimised to achieve better prediction accuracy for a particular forecast horizon. In addition, the confidence interval computed by the GP/CoRGP may be used by the MPC to perform stochastic optimisation. These suggestions may be included in future work.

Fig. 6 shows the computed $\mathrm{CoE}$ from experiments for various different scenarios. One key observation is that for the considered horizons, the CoRGP scenarios resulted in lower $\mathrm{CoE}$ than the GP counterpart. This is mainly attributed to a better prediction accuracy. The total $\mathrm{CoE}$ savings over one day for the CoRGP scenarios compared to GP scenarios vary approximately $1.3 \%$ to $7.7 \%$. This may appear to be small, but the accumulated savings over the longer term could be substantial. Interestingly, the four-hour CoRGP performed more superior than the six-hour GP case. Certainly, the offline scenario with perfect PV power and load demand forecasts produced the lowest CoE. However, it is worth mentioning that this is impractical for real implementation as the computational time for twenty-four hours horizon is significant whilst the prediction accuracy is not guaranteed.

Next, we analyse the operation of the microgrids through the measured battery SoC profiles, as shown in Fig. 7. As the horizon increased, the EMS decided to charge the batteries earlier to take advantage of the cheaper CoE from the utility, or storing excess PV generation. Since the offline strategy has perfect input information, the optimisation was performed once and the corresponding charging/discharging solutions were executed in sequence with no corrective measure taken to ensure the battery SoC reached the ideal values computed by the EMS. As a result, deviations from the battery SoC profiles

TABLE II

ERROR COMPARISON BETWEEN GP AND CORGP

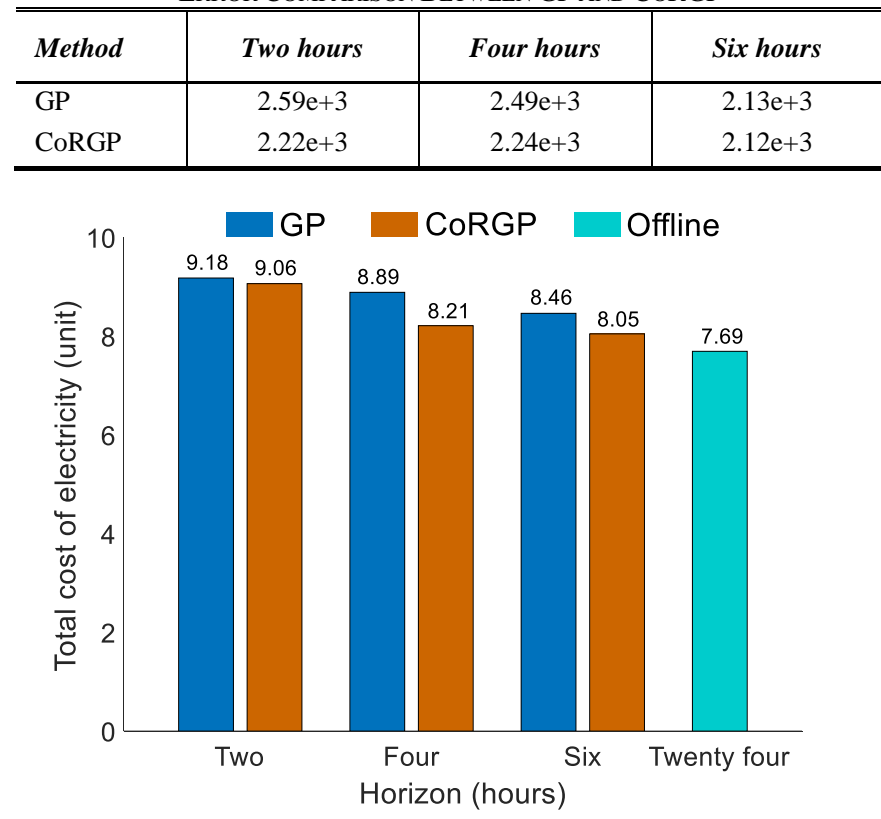

Fig. 6. Comparison of cost of electricity for different scenarios 

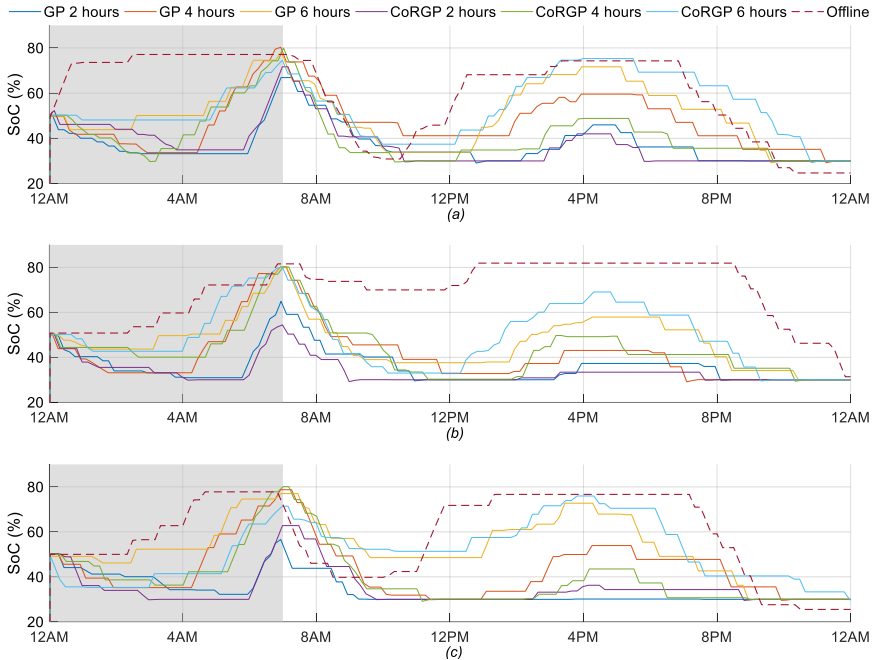

Fig. 7. Battery cycling profiles for (a) microgrid 1, (b) microgrid 2 and (c) microgrid 3. Time horizons of 2, 4 and 6 hours for GP and CoRGP cases along with offline approach are demonstrated

were observed, and at certain times, the SoC exceeded the limits or did not achieve the SoC targets due to the discrepancies between the MPC model and the hardware system. For instance, at around 7 am, all microgrids were targeted for $80 \%$ SoC but discrepancies can be noticed, e.g. microgrid 1 and 3 slightly below whilst microgrid 2 slightly above $80 \%$.

Although longer horizons produce improved $\mathrm{CoE}$ for the consumers, this may come at the expense of battery lifetime due to the higher cycling rates. One of the contributors to the described discrepancy is the presence of losses in the experimental setup, which were not accurately modelled in the EMS. Since microgrid 2 is directly connected to the main grid which controls the bus voltage, the voltage drop within that microgrid is minimal compared to the downstream microgrids 1 and 3. Due to the higher voltage drops experienced by the latter, the batteries must provide more current to achieve the same power output. As a result, for a day's operation, the battery SoC may be $5 \%$ lower than expected, depending on the distance of a particular microgrid from the slack bus.

\section{CONCLUSION}

This paper proposed and demonstrated the working principles of GP-MPC algorithm in a networked microgrid system. The objective was to quantify the trade-off and value gained by having more accurate renewable and load forecasts through the use of data from nearby regions, compared to the case of locally performed predictions. First, the modelling of GP and MPC algorithms along with their constraints was given. Using our laboratory-scaled microgrid system, experimental tests were conducted to study the relative savings on $\mathrm{CoE}$ one may achieve (about $1.3 \%$ to $7.7 \%$ ) by having a more accurate prediction. Besides computationally demanding, an offline (24hour horizon, perfect foresight) EMS strategy poses the risk of not being able to update the system states when deviations occur in reality. This could be fixed, but would be computationally expensive. Future work might consider understanding the relationship between battery sizing and the potential CoE savings. This may require a long-term economic study and proper characterisation of cost associated with battery cycling.

\section{REFERENCES}

[1] K. Rahbar, C. C. Chai, and R. Zhang, "Energy Cooperation Optimization in Microgrids With Renewable Energy Integration," IEEE Transactions on Smart Grid, vol. 9, no. 2, pp. 1482-1493, 2018.

[2] Y. Zhang, R. Wang, T. Zhang, Y. Liu, and B. Guo, "Model predictive control-based operation management for a residential microgrid with considering forecast uncertainties and demand response strategies," IET Generation, Transmission \& Distribution, vol. 10, no. 10, pp. 23672378, 2016.

[3] T. Alnejaili, S. Drid, D. Mehdi, L. Chrifi-Alaoui, R. Belarbi, and A. Hamdouni, "Dynamic control and advanced load management of a standalone hybrid renewable power system for remote housing," Energy Conversion and Management, vol. 105, pp. 377-392, 2015.

[4] A. Tani, M. B. Camara, and B. Dakyo, "Energy Management in the Decentralized Generation Systems Based on Renewable EnergyUltracapacitors and Battery to Compensate the Wind/Load Power Fluctuations," IEEE Transactions on Industry Applications, vol. 51, no. 2, pp. 1817-1827, 2015.

[5] A. Parisio, C. Wiezorek, T. Kyntäjä, J. Elo, K. Strunz, and K. H. Johansson, "Cooperative MPC-Based Energy Management for Networked Microgrids," IEEE Transactions on Smart Grid, vol. 8, no. 6, pp. 3066-3074, 2017.

[6] S. R. Cominesi, M. Farina, L. Giulioni, B. Picasso, and R. Scattolini, "A Two-Layer Stochastic Model Predictive Control Scheme for Microgrids," IEEE Transactions on Control Systems Technology, vol. 26, no. 1, pp. 1-13, 2018.

[7] B. Zhao, X. Wang, D. Lin, M. Calvin, J. Morgan, R. Qin, and C. Wang, "Energy Management of Multiple-Microgrids based on a System of Systems Architecture," IEEE Transactions on Power Systems, vol. 33, no. 6, pp. 6410-6421, 2018.

[8] A. M. Jadhav and N. R. Patne, "Priority-Based Energy Scheduling in a Smart Distributed Network With Multiple Microgrids," IEEE Transactions on Industrial Informatics, vol. 13, no. 6, pp. 3134-3143, 2017.

[9] A. C. Luna, L. Meng, N. L. Diaz, M. Graells, J. C. Vasquez, and J. M. Guerrero, "Online Energy Management Systems for Microgrids: Experimental Validation and Assessment Framework," IEEE Transactions on Power Electronics, vol. 33, no. 3, pp. 2201-2215, 2018.

[10] K. Rahbar, J. Xu, and R. Zhang, "Real-Time Energy Storage Management for Renewable Integration in Microgrid: An Off-Line Optimization Approach," IEEE Transactions on Smart Grid, vol. 6, no. 1, pp. 124-134, 2015.

[11] A. Parisio, E. Rikos, and L. Glielmo, "A Model Predictive Control Approach to Microgrid Operation Optimization," IEEE Transactions on Control Systems Technology, vol. 22, no. 5, pp. 1813-1827, 2014.

[12] R. Palma-Behnke, C. Benavides, F. Lanas, B. Severino, L. Reyes, J. Llanos, and D. Sáez, "A Microgrid Energy Management System Based on the Rolling Horizon Strategy," IEEE Transactions on Smart Grid, vol. 4, no. 2, pp. 996-1006, 2013.

[13] D. Ganger, J. Zhang, and V. Vittal, "Forecast-Based Anticipatory Frequency Control in Power Systems," IEEE Transactions on Power Systems, vol. 33, no. 1, pp. 1004-1012, 2018.

[14] F. Garcia-Torres and C. Bordons, "Optimal Economical Schedule of Hydrogen-Based Microgrids With Hybrid Storage Using Model Predictive Control," IEEE Transactions on Industrial Electronics, vol. 62, no. 8, pp. 5195-5207, 2015.

[15] F. Garcia-Torres, C. Bordons, and M. A. Ridao, "Optimal Economic Schedule for a Network of Microgrids with Hybrid Energy Storage System using Distributed Model Predictive Control," IEEE Transactions on Industrial Electronics, vol. 66, no. 3, pp. 1919-1929, 2019.

[16] Y. Du, J. Wu, S. Li, C. Long, and S. Onori, "Coordinated Energy Dispatch of Autonomous Microgrids With Distributed MPC Optimization," IEEE Transactions on Industrial Informatics, vol. 15, no. 9, pp. 5289-5298, 2019.

[17] D. Michaelson, H. Mahmood, and J. Jiang, "A Predictive Energy Management System Using Pre-Emptive Load Shedding for Islanded Photovoltaic Microgrids," IEEE Transactions on Industrial Electronics, vol. 64, no. 7, pp. 5440-5448, 2017.

[18] D. Romero-Quete and C. A. Cañizares, "An Affine Arithmetic-Based Energy Management System for Isolated Microgrids," IEEE Transactions on Smart Grid, vol. 10, no. 3, pp. 2989-2998, 2019.

[19] G. Mohy-Ud-Din, D. H. Vu, K. M. Muttaqi, and D. Sutanto, "An Integrated Energy Management Approach for the Economic Operation of Industrial Microgrids under Uncertainty of Renewable Energy," IEEE Transactions on Industry Applications, pp. 1-1, 2020. 
[20] L. Liang, Y. Hou, and D. J. Hill, "An Interconnected Microgrids-Based Transactive Energy System With Multiple Electric Springs," IEEE Transactions on Smart Grid, vol. 11, no. 1, pp. 184-193, 2020.

[21] Z. Liu, L. Wang, and L. Ma, "A Transactive Energy Framework for Coordinated Energy Management of Networked Microgrids With Distributionally Robust Optimization," IEEE Transactions on Power Systems, vol. 35, no. 1, pp. 395-404, 2020.

[22] M. Diagne, M. David, P. Lauret, J. Boland, and N. Schmutz, "Review of solar irradiance forecasting methods and a proposition for small-scale insular grids," Renewable and Sustainable Energy Reviews, vol. 27, pp. 65-76, 2013.

[23] R. Marquez and C. F. M. Coimbra, "Forecasting of global and direct solar irradiance using stochastic learning methods, ground experiments and the NWS database," Solar Energy, vol. 85, no. 5, pp. 746-756, 2011.

[24] S. Salcedo-Sanz, C. Casanova-Mateo, J. Muñoz-Marí, and G. CampsValls, "Prediction of Daily Global Solar Irradiation Using Temporal Gaussian Processes," IEEE Geoscience and Remote Sensing Letters, vol. 11, no. 11, pp. 1936-1940, 2014.

[25] D. Lee and R. Baldick, "Short-Term Wind Power Ensemble Prediction Based on Gaussian Processes and Neural Networks," IEEE Transactions on Smart Grid, vol. 5, no. 1, pp. 501-510, 2014.

[26] H. Sheng, J. Xiao, Y. Cheng, Q. Ni, and S. Wang, "Short-Term Solar Power Forecasting Based on Weighted Gaussian Process Regression," IEEE Transactions on Industrial Electronics, vol. 65, no. 1, pp. 300-308, 2018.

[27] C. Huang, L. Wang, and L. L. Lai, "Data-Driven Short-Term Solar Irradiance Forecasting Based on Information of Neighboring Sites," IEEE Transactions on Industrial Electronics, vol. 66, no. 12, pp. 99189927, 2019.

[28] L. K. Gan, A. Hussain, D. A. Howey, and H. Kim, "Limitations in Energy Management Systems: A Case Study for Resilient Interconnected Microgrids," IEEE Transactions on Smart Grid, vol. 10, no. 5, pp. 5675-5685, 2019.

[29] W. Shi, N. Li, C. C. Chu, and R. Gadh, "Real-Time Energy Management in Microgrids," IEEE Transactions on Smart Grid, vol. 8, no. 1, pp. 228238, 2017.

[30] D. S. Kirschen and G. Strbac, Fundamentals of Power System Economics, 1st ed.: Wiley, 2004.

[31] A. J. Wood, B. F. Wollenberg, and G. B. Sheblé, Power Generation, Operation and Control, 3rd ed.: Wiley-Blackwell, 2013.

[32] IBM, "IBM ILOG CPLEX Optimization Studio CPLEX User's Manual."

[33] E. Koubli, D. Palmer, T. Betts, P. Rowley, and R. Gottschalg, "Inference of missing PV monitoring data using neural networks," in 2016 IEEE 43rd Photovoltaic Specialists Conference (PVSC), 2016, pp. 3436-3440.

[34] C. E. Rasmussen and C. K. I. Williams, Gaussian Processes for Machine Learning: MIT Press, 2006.

[35] A. Mauricio A. and L. Neil D., "Sparse Convolved Multiple Output Gaussian Processes," arXiv:0911.5107, 2009.

[36] E.M.L. Beale and J. A. Tomlin, "Special facilities in a general mathematical programming system for non-convex problems using ordered sets of variables," in Proceedings of the fifth international conference on operational research, J.Lawrence, Tavistock Publications, London, 1970, pp. 447-454.

[37] L. K. Gan, B. Riar, J. Lee, and D. Howey, "Low-cost modular PV-battery microgrid emulator for testing of energy management algorithms," in 2017 IEEE Second International Conference on DC Microgrids (ICDCM), 2017, pp. 602-608.

[38] J. Lee, P. Zhang, L. K. Gan, D. A. Howey, M. A. Osborne, A. Tosi, and S. Duncan, "Optimal Operation of an Energy Management System Using Model Predictive Control and Gaussian Process Time-Series Modeling," IEEE Journal of Emerging and Selected Topics in Power Electronics, vol. 6, no. 4, pp. 1783-1795, 2018.

[39] Y. Du and F. Li, "A Hierarchical Real-Time Balancing Market Considering Multi-Microgrids With Distributed Sustainable Resources," IEEE Transactions on Sustainable Energy, vol. 11, no. 1, pp. 72-83, 2020.

[40] A. Ouammi, Y. Achour, D. Zejli, and H. Dagdougui, "Supervisory Model Predictive Control for Optimal Energy Management of Networked Smart Greenhouses Integrated Microgrid," IEEE Transactions on Automation Science and Engineering, vol. 17, no. 1, pp. $117-128,2020$.

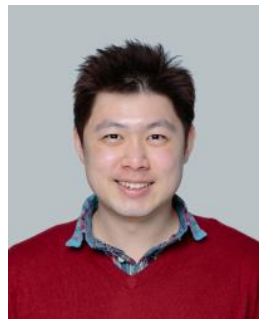

Leong Kit Gan (M'17) received the B.Eng. degree (Hons.) in electrical and electronics engineering from the Universiti Tenaga Nasional, Kuala Lumpur, Malaysia, in 2009, the M.Sc. degree in sustainable energy systems and the Ph.D. degree in energy systems from The University of Edinburgh, Edinburgh, U.K., in 2012 and 2016, respectively.

From 2016 to 2018, he was a Postdoctoral Researcher at the University of Oxford, focusing on intelligent control of decentralised energy systems. Between 2009 and 2011, he was an Electronics Engineer in Altera and Motorola.

His research interests include control of power electronics, energy storage and renewable energy systems.

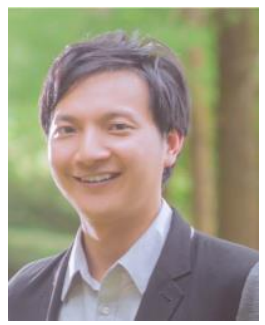

Pengfei Zhang received his Bachelor of Engineering in Electrical \& Electronic Engineering and Ph.D from Nanyang Technological University in 2010 and 2015 respectively. He is currently working at DBS bank as a data scientist. Between 2016 and 2018, he worked as a post-doctoral researcher at the Department of Engineering Science, University of Oxford. He was also a research scientist at the Institute for Infocomm research between 2014-2016 and 2018-2019. His research interests include gaussian process, energy efficient clustering algorithms, energy harvesting wireless sensor networks (EH-WSN), and statistical modeling in WSN.

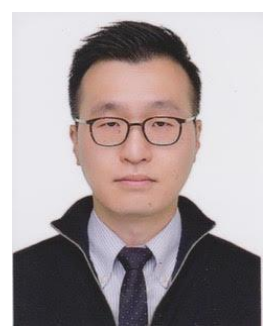

Jaehwa Lee received the bachelor's degree from the Pohang University of Science and Technology, Pohang, South Korea, the M.Sc. degree from Seoul National University, Seoul, South Korea, and the Ph.D. degree from the Control and Power Group, Imperial College London, U.K., in 2014.

He is currently a Senior Researcher with Samsung Advanced Institute of Technology, Suwon, South Korea. His research is focused on trajectory planning and decision making for autonomous vehicles.

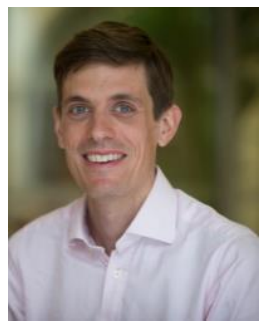

Mike Osborne is a Professor of Machine Learning in the Department of Engineering Science. He has acted as principal or co-investigator for $£ 10.6 \mathrm{M}$ of research funding. He has expertise in active learning, Gaussian processes, Bayesian optimisation, and Bayesian quadrature, and is a co-founder of the emerging field of probabilistic numerics. His algorithms have been applied in fields as diverse as astrostatistics, ornithology, and sensor networks. Mike is also the cofounder of the Oxford spin-out company Mind Foundry Ltd, which has grown to thirty employees (including eleven PhDs). Finally, Mike's work on the societal impacts of machine learning and robotics has been cited over 4000 times, and resulted in both sustained coverage in almost all major media venues (e.g. his being interviewed on BBC Newsnight, a cover feature in the Economist) and policy impact (including presenting oral evidence to the UK House of Commons Science and Technology Committee).

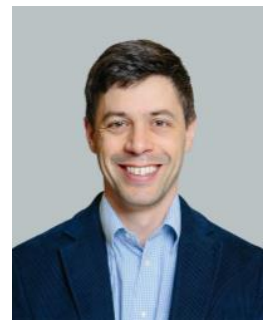

David A. Howey (M'10-SM'17) received the B.A. and M.Eng. degrees from Cambridge University, Cambridge, U.K., in 2002 and the Ph.D. degree from Imperial College London, London, U.K., in 2010. He is currently an Associate Professor at the Department of Engineering Science, University of Oxford, Oxford, U.K. His research is focused on energy storage systems, including projects on model-based battery management, degradation, thermal management, and energy management for grid 